\title{
Stabilizing Fuzzy Output Control for a Class of Nonlinear Systems
}

\author{
Dušan Krokavec and Anna Filasová \\ Department of Cybernetics and Artificial Intelligence, Faculty of Electrical Engineering and Informatics, \\ Technical University of Košice, Letná 9, 04200 Košice, Slovakia \\ Correspondence should be addressed to Dušan Krokavec; dusan.krokavec@tuke.sk
}

Received 27 September 2012; Revised 15 December 2012; Accepted 17 December 2012

Academic Editor: Sendren Sheng-Dong Xu

Copyright (c) 2013 D. Krokavec and A. Filasová. This is an open access article distributed under the Creative Commons Attribution License, which permits unrestricted use, distribution, and reproduction in any medium, provided the original work is properly cited.

The paper presents new conditions suitable in design of a stabilizing output controller for a class of continuous-time nonlinear systems, represented by Takagi-Sugeno models. Taking into account the affine properties of the TS model structure and applying the fuzzy control scheme relating to the parallel distributed output compensators, the sufficient design conditions are outlined in terms of linear matrix inequalities. The proposed procedure decouples the Lyapunov matrix and the system parameter matrices in the LMIs and guarantees global stability of the system. Simulation result illustrates the design procedure and demonstrates the performances of the proposed design method.

\section{Introduction}

Contrarily to the linear framework, nonlinear systems are too complex to be represented by unified mathematical resources, and so a generic method has not been developed yet to design a controller valid for all types of nonlinear systems. An alternative to nonlinear system models is TakagiSugeno (TS) fuzzy approach [1], which benefits from the advantages of suitable linear approximation. Using the TS fuzzy model, each rule utilizes the local system dynamics by a linear model and the nonlinear system is represented by a collection of fuzzy rules. Recently, TS model based fuzzy control approaches are being fast and successfully used in nonlinear control frameworks. As a result, a range of stability analysis conditions [2-5], as well as control design methods [6-11], have been developed for TS fuzzy systems, relying mostly on the feasibility of an associated set of linear matrix inequalities (LMI) [12]. An important fact is that the design problem is a standard feasibility problem with several LMIs, potentially reformulated such that the feedback gains can be solved numerically. In consequence, the state feedback control based on fuzzy TS systems model is mostly realized in such structures which can be designed using a technique based on LMIs.

The TS fuzzy model-based state control is based on an implicit assumption that all states are available for measurement. If it is impossible, TS fuzzy observers are used to estimate the unmeasurable states variables, and the state controller exploits the system state variable estimate values [13-15]. The nonlinear output feedback design is so formulated as the two LMI set problems, and treated by the two-stage procedure, that is, dealing with a set of LMIs for the observer parameters at first and then solving another set of LMIs for the controller parameters [16]. Since the fuzzy control design problem is preferred to be formulated as a one LMI set problem, such formulation for the output feedback control design is proposed in $[17,18]$.

The main contribution of the paper is the presentation of the original design condition of the fuzzy output feedback control for the continuous-time nonlinear MIMO systems approximated by a TS model. The central idea of the TS fuzzy model-based control design, that is, to derive control rules so as to compensate each rule of a fuzzy system and construct the control strategy based on the parallel distributed compensators, is reflected in the approach of output control, taking into account the fact that the desired output variables are measurable. Motivated by the above-mentioned observations, the proposed design method combines the principles given in $[15,17]$, respecting the results presented in [19], and is constructed on an enhanced form of quadratic Lyapunov function [20]. Comparing with the approaches based on a quadratic Lyapunov matrix $[15,17,21]$, which are particularly in the case of a large number of rules very conservative as a common symmetric positive definite matrix 
is used to verify all Lyapunov inequalities, presented principle naturally extends the affine TS model properties using slack matrix variables to decouple Lyapunov matrix and the system matrices in LMIs, does not use iterative algorithms based on LMIs or matrix norm bounds, and gives substantial reducing of conservativeness. Potentially, extra constraints can be imposed to the slack matrices, but such additive constraints can potentially increase the conservativeness of the proposed design conditions.

The remainder of this paper is organized as follows. In Section 2 the structure of TS model for considered class of nonlinear systems is briefly described, and some of its properties are outlined. The output feedback control design problem for systems with measurable premise variables is given in Section 3, where the design conditions that guarantee global quadratic stability are formulated and proven. The method is reformulated in Section 4 in a newly defined enhanced criterion for fuzzy output feedback control design. Section 5 gives the numerical example to illustrate the effectiveness of the proposed approach and to confirm the validity of the control scheme. The last section draws conclusions and some future directions.

Throughout the paper, the following notations are used: $\mathbf{x}^{T}, \mathbf{X}^{T}$ denote the transpose of the vector $\mathbf{x}$ and matrix $\mathbf{X}$, respectively, diag[.] denotes a block diagonal matrix, for a square matrix $\mathbf{X}=\mathbf{X}^{T}>0$ (resp., $\mathbf{X}=\mathbf{X}^{T}<0$ ) means that $\mathbf{X}$ is a symmetric positive definite matrix (resp., negative definite matrix), the symbol $\mathbf{I}_{n}$ represents the $n$th order unit matrix, $\mathbb{R}$ denotes the set of real numbers, and $\mathbb{R}^{n \times r}$ denotes the set of all $n \times r$ real matrices.

\section{Takagi-Sugeno Fuzzy Models}

The systems under consideration are from one class of multiinput and multioutput nonlinear (MIMO) dynamic systems, represented in state-space form as

$$
\begin{gathered}
\dot{\mathbf{q}}(t)=\mathbf{a}(\mathbf{q}(t))+\mathbf{b}(\mathbf{q}(t)) \mathbf{u}(t), \\
\mathbf{y}(t)=\mathbf{C q}(t)
\end{gathered}
$$

where $\mathbf{q}(t) \in \mathbb{R}^{n}, \mathbf{u}(t) \in \mathbb{R}^{r}$, and $\mathbf{y}(t) \in \mathbb{R}^{m}$ are vectors of the state, input, and output variables, $\mathbf{C} \in \mathbb{R}^{m \times n}$ is a real finite values matrix, and $m \leq n, r \leq n$, respectively.

Considering that the number of the nonlinear terms in $\mathbf{a}(\mathbf{q}(t))$ is $p$, there exists a set of nonlinear sector functions as follows:

$$
\begin{aligned}
m_{l j}\left(\theta_{j}(t)\right), \quad j & =1,2, \ldots, k, l=1,2, \ldots, p, \\
m_{l 1}\left(\theta_{j}(t)\right) & =1-\sum_{j=2}^{k} m_{l j}\left(\theta_{j}(t)\right)
\end{aligned}
$$

where $k$ is the number of sectors, and

$$
\boldsymbol{\theta}(t)=\left[\begin{array}{llll}
\theta_{1}(t) & \theta_{2}(t) & \cdots & \theta_{q}(t)
\end{array}\right]
$$

is the vector of premise variables. It is assumed that the premise variable is a system state variable, or a measurable external variable, and none of the premise variables does not depend on the inputs $\mathbf{u}(t)$.

Using a TS model, the conclusion part of a single rule consists no longer of a fuzzy set [3], but determines a function with state variables as arguments, and the corresponding function is a local function for the fuzzy region that is described by the premise part of the rule. Thus, using linear functions, a system state is described locally (in fuzzy regions) by linear models, and at the boundaries between regions an interpolation is used between the corresponding local models.

Thus, constructing the set of membership functions $\left\{w_{i}(\boldsymbol{\theta}(t))=\prod_{j=1}^{s} m_{l j}\left(\theta_{j}(t)\right), i=1,2, \ldots s, s=2^{k}\right\}$ from all combinations of sector functions, the final states of the systems are inferred as follows:

$$
\dot{\mathbf{q}}(t)=\sum_{i=1}^{s} h_{i}(\boldsymbol{\theta}(t))\left(\mathbf{A}_{i} \mathbf{q}(t)+\mathbf{B}_{i} \mathbf{u}(t)\right)
$$

with the output given by the relation

$$
\mathbf{y}(t)=\mathbf{C q}(t),
$$

where

$$
h_{i}(\boldsymbol{\theta}(t))=\frac{w_{i}(\boldsymbol{\theta}(t))}{\sum_{i=1}^{s} w_{i}(\boldsymbol{\theta}(t))}
$$

is the average weight for the $i$ th rule, representing the normalized grade of membership (membership function). By definition, the membership functions satisfy the following convex sum properties:

$$
0 \leq h_{i}(\boldsymbol{\theta}(t)) \leq 1, \quad \sum_{i=1}^{s} h_{i}(\boldsymbol{\theta}(t))=1 \quad \forall i \in\langle 1, \ldots, s\rangle
$$

Assuming that $\mathbf{a}(\mathbf{q}(t))$ and $\mathbf{b}(\mathbf{q}(t))$ are bounded in sectors, that is, in the fuzzy regions within the system will operate, and $\mathbf{a}(\mathbf{q}(t))$ takes the value $\mathbf{a}(\mathbf{0})=\mathbf{0}$, the fuzzy approximation of (1) leads to (6). Thus, $\mathbf{A}_{i} \in \mathbb{R}^{n \times n}$ is the matrix of $\mathbf{a}(\mathbf{p}(t)), \mathbf{B}_{i} \in$ $\mathbb{R}^{n \times r}$ is the matrix of $\mathbf{b}(\mathbf{p}(t))$, respectively, both for $\mathbf{p}(t)=\mathbf{p}_{i}$, where $\mathbf{p}_{i}$ is the $i$ th combination of the bounds of the sector functions with respect to the center of the $i$ th fuzzy region, dedicated by (3). It is evident that a general fuzzy model is achieved by fuzzy amalgamation of the linear systems models.

Note, the model (5) and (6) is mostly considered for analysis, control, and state estimation of nonlinear systems.

Assumption 1. Each triplet $\left(\mathbf{A}_{i}, \mathbf{B}_{i}, \mathbf{C}\right)$ is locally controllable and observable, the matrix $\mathbf{C}$ is the same for all local models, and the number of input variables is equal to the number of output variables.

It is supposed in the following considerations that the aforementioned model does not include parameter uncertainties or external disturbances, and the premise variables are measured. 


\section{Fuzzy Output Control Design}

In the next, the fuzzy output controller is designed using the concept of parallel distributed compensation, in which the fuzzy controller shares the same sets of normalized membership functions like the TS fuzzy system model.

Definition 1. Considering (5) and (6), and using the same set of normalized membership function (8), the fuzzy static output controller is defined as

$$
\mathbf{u}(t)=-\sum_{j=1}^{s} h_{j}(\boldsymbol{\theta}(t)) \mathbf{K}_{\mathbf{j}} \mathbf{y}(t)=-\sum_{j=1}^{s} h_{j}(\boldsymbol{\theta}(t)) \mathbf{K}_{j} \mathbf{C q}(t) .
$$

Note that the fuzzy controller (9) is in general nonlinear.

Theorem 2. The equilibrium of the fuzzy system (5) and (6), controlled by the fuzzy controller (9), is global asymptotically stable if there exist a positive definite symmetric matrix $\mathbf{W} \in$ $\mathbb{R}^{n \times n}$ and matrices $\mathbf{N}_{j}, \mathbf{Y}_{i j} \in \mathbb{R}^{r \times n}$ such that

$$
\mathbf{W}=\mathbf{W}^{T}>0, \quad\left[\begin{array}{cccc}
\mathbf{Y}_{11} & \mathbf{Y}_{12} & \cdots & \mathbf{Y}_{1 s} \\
\mathbf{Y}_{12} & \mathbf{Y}_{22} & \cdots & \mathbf{Y}_{2 s} \\
\vdots & \vdots & \ddots & \vdots \\
\mathbf{Y}_{1 s} & \mathbf{Y}_{2 s} & \cdots & \mathbf{Y}_{s s}
\end{array}\right]>0
$$

$$
\mathbf{A}_{i} \mathbf{W}+\mathbf{W A}_{i}^{T}-\mathbf{B}_{i} \mathbf{N}_{j} \mathbf{C}-\mathbf{C}^{T} \mathbf{N}_{j}^{T} \mathbf{B}_{i}^{T}+\mathbf{Y}_{i j}<0
$$

for $h_{i}(\boldsymbol{\theta}(t)) h_{j}(\boldsymbol{\theta}(t)) \neq 0, i, j=1,2, \ldots, s$.

If the above conditions hold, the set of control law gain matrices is given as

$$
\mathbf{K}_{j}=\mathbf{N}_{j} \mathbf{M}^{-1}, \quad j=1,2, \ldots, s,
$$

where

$$
\mathbf{M}=\mathbf{C W} \mathbf{C}^{\ominus 1}, \quad \mathbf{C}^{\ominus 1}=\mathbf{C}^{T}\left(\mathbf{C C}^{T}\right)^{-1} .
$$

$\mathbf{C}^{\ominus 1}$ is Moore-Penrose pseudo-inverse of $\mathbf{C}$.

Proof. Considering the model (6) and (2) and the control law (9), then it yields

$$
\dot{\mathbf{q}}(t)=\sum_{i=1}^{s} \sum_{j=1}^{s} h_{i}(\boldsymbol{\theta}(t)) h_{j}(\boldsymbol{\theta}(t))\left(\mathbf{A}_{i}-\mathbf{B}_{i} \mathbf{K}_{j} \mathbf{C}\right) \mathbf{q}(t) .
$$

In order to analyze the convergence of the system state, the quadratic positive Lyapunov function is considered as follows:

$$
v(\mathbf{q}(t))=\mathbf{q}^{T}(t) \mathbf{P q}(t)>0,
$$

where $\mathbf{P} \in \mathbb{R}^{n \times n}$ is a positive definite symmetric matrix. Then, the time derivative of $v(\mathbf{q}(t))$ along the system trajectory is

$$
\dot{v}(\mathbf{q}(t))=\dot{\mathbf{q}}^{T}(t) \mathbf{P q}(t)+\mathbf{q}^{T}(t) \mathbf{P} \dot{\mathbf{q}}(t)<0 .
$$

Substituting (14) in (16), and introducing the term as follows:

$$
v_{v}(\boldsymbol{\theta}(t))=\mathbf{q}^{T}(t) \mathbf{Z}(\boldsymbol{\theta}(t)) \mathbf{q}(t),
$$

where

$$
\mathbf{Z}(\boldsymbol{\theta}(t))=\sum_{i=1}^{s} \sum_{j=1}^{s} h_{i}(\boldsymbol{\theta}(t)) h_{j}(\boldsymbol{\theta}(t)) \mathbf{X}_{i j}>0
$$

and $\left\{\mathbf{X}_{i j}=\mathbf{X}_{j i}^{T} \in \mathbb{R}^{n \times n}, i, j=1,2, \ldots, s\right\}$ is the set of matrices. In the sense of Krasovskii theorem (see, e.g., [22]) it can be set up as follows:

$$
\begin{aligned}
& \dot{v}(\mathbf{q}(t)) \\
&= \mathbf{q}^{T}(t) \sum_{i=1}^{s} \sum_{j=1}^{s} h_{i}(\boldsymbol{\theta}(t)) h_{j}(\boldsymbol{\theta}(t)) \mathbf{P}\left(\mathbf{A}_{i}-\mathbf{B}_{i} \mathbf{K}_{j} \mathbf{C}\right) \mathbf{q}(t) \\
&+\mathbf{q}^{T}(t) \sum_{i=1}^{s} \sum_{j=1}^{s} h_{i}(\boldsymbol{\theta}(t)) h_{j}(\boldsymbol{\theta}(t)) \\
& \times\left(\mathbf{A}_{i}-\mathbf{B}_{i} \mathbf{K}_{j} \mathbf{C}\right)^{T} \mathbf{P q}(t) \\
&<-\mathbf{q}^{T}(t) \sum_{i=1}^{s} \sum_{j=1}^{s} h_{i}(\boldsymbol{\theta}(t)) h_{j}(\boldsymbol{\theta}(t)) \mathbf{X}_{i j} \mathbf{q}(t) \\
&=-\mathbf{q}^{T}(t) \mathbf{Z}(\boldsymbol{\theta}(t)) \mathbf{q}(t)<0, \\
& \dot{v}(\mathbf{q}(t))=\sum_{i=1}^{s} \sum_{j=1}^{s} h_{i}(\boldsymbol{\theta}(t)) h_{j}(\boldsymbol{\theta}(t)) \mathbf{q}^{T}(t) \mathbf{P}_{i j} \mathbf{q}(t)<0
\end{aligned}
$$

respectively, which implies

$$
\mathbf{P}_{i j}=\mathbf{P}\left(\mathbf{A}_{i}-\mathbf{B}_{i} \mathbf{K}_{j} \mathbf{C}\right)+\left(\mathbf{A}_{i}-\mathbf{B}_{i} \mathbf{K}_{j} \mathbf{C}\right)^{T} \mathbf{P}+\mathbf{X}_{i j}<0 .
$$

It is evident that $\mathbf{P}_{i j}$ has to be negative definite.

Since $r=m$, it is possible to set

$$
\mathbf{B}_{i} \mathbf{K}_{j} \mathbf{C}=\mathbf{B}_{i} \mathbf{K}_{j} \mathbf{M M}^{-1} \mathbf{C}=\mathbf{B}_{i} \mathbf{N}_{j} \mathbf{C P},
$$

where

$$
\mathbf{K}_{j} \mathbf{M}=\mathbf{N}_{j}, \quad \mathbf{M}^{-1} \mathbf{C}=\mathbf{C P}
$$

and $\mathbf{M} \in \mathbb{R}^{m \times m}$ is a regular square matrix. Substituting (22) into (21) results in

$$
\mathbf{P}\left(\mathbf{A}_{i}-\mathbf{B}_{i} \mathbf{N}_{j} \mathbf{C P}\right)+\left(\mathbf{A}_{i}-\mathbf{B}_{i} \mathbf{N}_{j} \mathbf{C P}\right)^{T} \mathbf{P}+\mathbf{X}_{i j}<0 .
$$

Premultiplying the left side and the right side of (24) by $\mathbf{P}^{-1}$ leads to

$$
\mathbf{A}_{i} \mathbf{P}^{-1}-\mathbf{B}_{i} \mathbf{N}_{j} \mathbf{C}+\mathbf{P}^{-1} \mathbf{A}_{i}^{T}-\mathbf{C}^{T} \mathbf{N}_{j}^{T} \mathbf{B}_{i}^{T}+\mathbf{P}^{-1} \mathbf{X}_{i j} \mathbf{P}^{-1}<0
$$

and using the notations

$$
\mathbf{W}=\mathbf{P}^{-1}, \quad \mathbf{Y}_{i j}=\mathbf{W} \mathbf{X}_{i j} \mathbf{W}
$$

then (25) implies (11). Since (25) also implies

$$
\widetilde{\mathbf{P}}_{i j}=\mathbf{A}_{i} \mathbf{W}+\mathbf{W A}_{i}^{T}-\mathbf{B}_{i} \mathbf{N}_{j} \mathbf{C}-\mathbf{C}^{T} \mathbf{N}_{j}^{T} \mathbf{B}_{i}^{T}+\mathbf{Y}_{i j}<0 .
$$


Using the membership functions property (8) and defining $\mathbf{q}^{\diamond}(t)=\mathbf{W}^{-1} \mathbf{q}(t)$, it can be written as follows:

$$
\begin{aligned}
\dot{v}_{v} & (\mathbf{q}(t)) \\
& =\sum_{i=1}^{s} \sum_{j=1}^{s} h_{i}(\boldsymbol{\theta}(t)) h_{j}(\boldsymbol{\theta}(t)) \mathbf{q}^{T}(t) \mathbf{W}^{-1} \mathbf{W} \mathbf{X}_{i j} \mathbf{W} \mathbf{W}^{-1} \mathbf{q}(t) \\
& =\sum_{i=1}^{s} \sum_{j=1}^{s} h_{i}(\boldsymbol{\theta}(t)) h_{j}(\boldsymbol{\theta}(t)) \mathbf{q}^{\diamond T}(t) \mathbf{Y}_{i j} \mathbf{q}^{\diamond}(t) \\
& =\mathbf{q}^{\diamond T}(t) \mathbf{W Z}(\boldsymbol{\theta}(t)) \mathbf{W} \mathbf{q}^{\diamond}(t)>0 .
\end{aligned}
$$

Writing $\mathbf{Z}(\boldsymbol{\theta}(t))$ as follows:

$$
\begin{aligned}
\mathbf{Z}(\boldsymbol{\theta}(t))= & {\left[\begin{array}{c}
h_{1}(\boldsymbol{\theta}(t)) \mathbf{q}^{\diamond}(t) \\
h_{2}(\boldsymbol{\theta}(t)) \mathbf{q}^{\diamond}(t) \\
\vdots \\
h_{s}(\boldsymbol{\theta}(t)) \mathbf{q}^{\diamond}(t)
\end{array}\right]^{T}\left[\begin{array}{cccc}
\mathbf{Y}_{11} & \mathbf{Y}_{12} & \cdots & \mathbf{Y}_{1 s} \\
\mathbf{Y}_{21} & \mathbf{Y}_{22} & \cdots & \mathbf{Y}_{2 s} \\
\vdots & \vdots & \ddots & \vdots \\
\mathbf{Y}_{s 1} & \mathbf{Y}_{s 2} & \cdots & \mathbf{Y}_{s s}
\end{array}\right] } \\
& \times\left[\begin{array}{c}
h_{1}(\boldsymbol{\theta}(t)) \mathbf{q}^{\diamond}(t) \\
h_{2}(\boldsymbol{\theta}(t)) \mathbf{q}^{\diamond}(t) \\
\vdots \\
h_{s}(\boldsymbol{\theta}(t)) \mathbf{q}^{\diamond}(t)
\end{array}\right]>0
\end{aligned}
$$

then (29) implies (10). In addition, (23) gives

$$
\mathbf{M C}=\mathbf{C P}^{-1}=\mathbf{C W} .
$$

Premultiplying the right side of (30) by $\mathbf{C}^{T}$ gives

$$
\mathbf{C W C}^{T}=\mathbf{M C C}^{T}
$$

and so it is

$$
\mathbf{M}=\mathbf{C W C}^{T}\left(\mathbf{C C}^{T}\right)^{-1}=\mathbf{C W C}^{\ominus 1}
$$

Thus, (23) and (32) imply (12)-(13), respectively. This concludes the proof.

Note, the derived results are linked to some existing finding when the design problem involves additive performance requirements and the relaxed quadratic stability conditions of fuzzy control systems (see, e.g., $[13,23]$ ) are equivalently steered.

Trying to minimize the number of LMIs owing to the limitation of solvers, Theorem 2 can be presented in the equivalent structure in which the number of stabilization conditions, used in fuzzy controller design, is equal to $N=$ $\left(s^{2}+s\right) / 2+1$. Evidently, the number of stabilization conditions is substantially reduced if $s$ is large.

Theorem 3. The equilibrium of the fuzzy system (5) and (6), controlled by the fuzzy controller (9), is global asymptotically stable if there exist a positive definite symmetric matrix $\mathbf{W} \in$ $\mathbb{R}^{n \times n}$ and matrices $\mathbf{N}_{j}, \mathbf{Y}_{i j} \in \mathbb{R}^{r \times n}$ such that

$$
\begin{gathered}
\mathbf{W}=\mathbf{W}^{T}>0, \quad\left[\begin{array}{cccc}
\mathbf{Y}_{11} & \mathbf{Y}_{12} & \cdots & \mathbf{Y}_{1 s} \\
\mathbf{Y}_{21} & \mathbf{Y}_{22} & \cdots & \mathbf{Y}_{2 s} \\
\vdots & \vdots & \ddots & \vdots \\
\mathbf{Y}_{s 1} & \mathbf{Y}_{s 2} & \cdots & \mathbf{Y}_{s s}
\end{array}\right]>0 \\
\mathbf{H}_{i i}+\mathbf{H}_{i i}^{T}+\mathbf{Y}_{i i}<0, \\
\frac{\mathbf{H}_{i j}+\mathbf{H}_{j i}}{2}+\frac{\mathbf{H}_{i j}^{T}+\mathbf{H}_{j i}^{T}}{2}+\frac{\mathbf{Y}_{i j}+\mathbf{Y}_{j i}}{2}<0
\end{gathered}
$$

for all $i \in\langle 1,2, \ldots, s\rangle, i<j \leq s, i, j \in\langle 1,2, \ldots, s\rangle$, respectively, and $h_{i}(\boldsymbol{\theta}(t)) h_{j}(\boldsymbol{\theta}(t)) \neq 0$. Here it is

$$
\mathbf{H}_{i j}=\mathbf{A}_{i} \mathbf{W}-\mathbf{B}_{i} \mathbf{N}_{j} \mathbf{C}
$$

and if the above conditions hold, the set of control law gain matrices is given as in (12) and (13).

Proof. Now (28) can be written as

$$
\begin{aligned}
\dot{v}(\mathbf{q}(t))= & \sum_{i=1}^{s} \sum_{j=1}^{s} h_{i}(\boldsymbol{\theta}(t)) h_{j}(\boldsymbol{\theta}(t)) \mathbf{q}^{T}(t) \\
& \times\left(\mathbf{H}_{i j}+\mathbf{H}_{i j}^{T}+\mathbf{Y}_{i j}\right) \mathbf{q}(t),
\end{aligned}
$$

where $\mathbf{H}_{i j}$ is given in (35). Permuting the subscripts $i$ and $j$ in (36) gives

$$
\begin{aligned}
\dot{v}(\mathbf{q}(t))= & \sum_{i=1}^{s} \sum_{j=1}^{s} h_{i}(\boldsymbol{\theta}(t)) h_{j}(\boldsymbol{\theta}(t)) \mathbf{q}^{T}(t) \\
& \times\left(\mathbf{H}_{j i}+\mathbf{H}_{j i}^{T}+\mathbf{Y}_{j i}\right) \mathbf{q}(t)
\end{aligned}
$$

and adding (36) and (37) results in

$$
2 \dot{v}(\mathbf{q}(t))
$$

$$
\begin{aligned}
= & \sum_{i=1}^{s} \sum_{j=1}^{s} h_{i}(\boldsymbol{\theta}(t)) h_{j}(\boldsymbol{\theta}(t)) \mathbf{q}^{T}(t) \\
& \times\left(\mathbf{H}_{i j}+\mathbf{H}_{j i}+\left(\mathbf{H}_{i j}+\mathbf{H}_{j i}\right)^{T}+\left(\mathbf{Y}_{i j}+\mathbf{Y}_{j i}\right)\right) \mathbf{q}(t) .
\end{aligned}
$$

Rearranging the computation, (38) takes the following form:

$$
\begin{aligned}
\dot{v}(\mathbf{q}(t))= & \sum_{i=1}^{s} h_{i}^{2}(\boldsymbol{\theta}(t)) \mathbf{q}^{T}(t)\left(\mathbf{H}_{i i}+\mathbf{H}_{i i}^{T}-\mathbf{Y}_{i i}\right) \mathbf{q}(t) \\
& +2 \sum_{i=1}^{s-1} \sum_{j=i+1}^{s} h_{i}(\boldsymbol{\theta}(t)) h_{j}(\boldsymbol{\theta}(t)) \mathbf{q}^{T}(t) \\
& \times\left(\frac{\mathbf{H}_{i j}+\mathbf{H}_{j i}}{2}+\frac{\mathbf{H}_{i j}^{T}+\mathbf{H}_{j i}^{T}}{2}+\frac{\mathbf{Y}_{i j}+\mathbf{Y}_{j i}}{2}\right) \mathbf{q}(t)
\end{aligned}
$$

and, evidently, (39) implies (34). This concludes the proof. 


\section{Enhanced Criterion for Output Control Design}

Theorem 4. The equilibrium of the fuzzy system (5) and (6), controlled by the fuzzy controller (9) is global asymptotically stable if there exist positive definite symmetric matrices $\mathbf{U}, \mathbf{V} \in$ $\mathbb{R}^{n \times n}$, a matrix $\mathbf{T} \in \mathbb{R}^{n \times n}$, and matrices $\mathbf{N}_{j}, \mathbf{Y}_{i j} \in \mathbb{R}^{r \times n}$ such that

$$
\begin{gathered}
\mathbf{U}=\mathbf{U}^{T}>0, \quad \mathbf{V}=\mathbf{V}^{T}>0 \\
{\left[\begin{array}{cccc}
\mathbf{Y}_{11} & \mathbf{Y}_{12} & \cdots & \mathbf{Y}_{1 s} \\
\mathbf{Y}_{12} & \mathbf{Y}_{22} & \cdots & \mathbf{Y}_{2 s} \\
\vdots & \vdots & \ddots & \vdots \\
\mathbf{Y}_{1 s} & \mathbf{Y}_{2 s} & \cdots & \mathbf{Y}_{s s}
\end{array}\right]>0,} \\
\mathbf{P}_{i j}^{\bullet}=\left[\begin{array}{ccc}
\mathbf{A}_{i} \mathbf{V}+\mathbf{V} \mathbf{A}_{i}^{T}-\mathbf{B}_{i} \mathbf{N}_{j} \mathbf{C}-\mathbf{C}^{T} \mathbf{N}_{j}^{T} \mathbf{B}_{i}^{T}+\mathbf{Y}_{i j} & * \\
\mathbf{T}-\mathbf{U}+\mathbf{A}_{i} \mathbf{V}-\mathbf{B}_{i} \mathbf{N}_{j} \mathbf{C} & -2 \mathbf{U}
\end{array}\right]<0
\end{gathered}
$$

for $h_{i}(\boldsymbol{\theta}(t)) h_{j}(\boldsymbol{\theta}(t)) \neq 0, i, j=1,2, \ldots, s$.

If the above conditions hold, the set of control law gain matrices is given as

$$
\mathbf{K}_{j}=\mathbf{N}_{j} \mathbf{M}^{-1}, \quad j=1,2, \ldots, s,
$$

where

$$
\mathbf{M}=\mathbf{C V C}^{\ominus 1}, \quad \mathbf{C}^{\ominus 1}=\mathbf{C}^{T}\left(\mathbf{C C}^{T}\right)^{-1},
$$

$\mathbf{C}^{\ominus 1}$ is Moore-Penrose pseudo-inverse of $\mathbf{C}$.

Here, and hereafter, * denotes the symmetric item in a symmetric matrix.

Proof. Writing (14) in the following form:

$$
\dot{\mathbf{q}}(t)-\sum_{i=1}^{s} \sum_{j=1}^{s} h_{i}(\boldsymbol{\theta}(t)) h_{j}(\boldsymbol{\theta}(t))\left(\mathbf{A}_{i}-\mathbf{B}_{i} \mathbf{K}_{j} \mathbf{C}\right) \mathbf{q}(t)=0
$$

then with arbitrary symmetric regular matrices $\mathbf{S}_{1}, \mathbf{S}_{2} \in \mathbb{R}^{n \times n}$ it yields

$$
\begin{aligned}
- & \left(\mathbf{q}^{T}(t) \mathbf{S}_{1}+\dot{\mathbf{q}}^{T}(t) \mathbf{S}_{2}\right) \\
& \times\left(\dot{\mathbf{q}}(t)-\sum_{i=1}^{s} \sum_{j=1}^{s} h_{i}(\boldsymbol{\theta}(t)) h_{j}(\boldsymbol{\theta}(t))\left(\mathbf{A}_{i}-\mathbf{B}_{i} \mathbf{K}_{j} \mathbf{C}\right) \mathbf{q}(t)\right)=0 .
\end{aligned}
$$

Thus, adding (45) as well as the transposition of (45) to (19), it yields

$$
\begin{aligned}
\dot{v}(\mathbf{q}(t))= & \dot{\mathbf{q}}^{T}(t) \mathbf{P} \mathbf{q}(t)+\mathbf{q}^{T}(t) \mathbf{P} \dot{\mathbf{q}}(t) \\
- & \left(\mathbf{q}^{T}(t) \mathbf{S}_{1}+\dot{\mathbf{q}}^{T}(t) \mathbf{S}_{2}\right) \\
& \times\left(\dot{\mathbf{q}}(t)-\sum_{i=1}^{s} \sum_{j=1}^{s} h_{i}(\boldsymbol{\theta}(t)) h_{j}(\boldsymbol{\theta}(t))\right. \\
& \left.\times\left(\mathbf{A}_{i}-\mathbf{B}_{i} \mathbf{K}_{j} \mathbf{C}\right) \mathbf{q}(t)\right) \\
& -\left(\dot{\mathbf{q}}^{T}(t)-\mathbf{q}^{T}(t) \sum_{i=1}^{s} \sum_{j=1}^{s} h_{i}(\boldsymbol{\theta}(t)) h_{j}(\boldsymbol{\theta}(t))\right. \\
& \left.\times\left(\mathbf{A}_{i}-\mathbf{B}_{i} \mathbf{K}_{j} \mathbf{C}\right)^{T}\right)\left(\mathbf{S}_{1} \mathbf{q}(t)+\mathbf{S}_{2} \dot{\mathbf{q}}(t)\right) \\
< & -\mathbf{q}^{T}(t) \sum_{i=1}^{s} \sum_{j=1}^{s} h_{i}(\boldsymbol{\theta}(t)) h_{j}(\boldsymbol{\theta}(t)) \mathbf{X}_{i j} \mathbf{q}(t) \\
=- & \mathbf{q}^{T}(t) \mathbf{Z}(\boldsymbol{\theta}(t)) \mathbf{q}(t)<0 .
\end{aligned}
$$

Using the notation

$$
\mathbf{q}^{\circ T}(t)=\left[\begin{array}{lll}
\mathbf{q}^{T}(t) & \dot{\mathbf{q}}^{T}(t)
\end{array}\right]
$$

the inequality (46) can be set and written as

$$
\dot{v}(\mathbf{q}(t))=\sum_{i=1}^{s} \sum_{j=1}^{s} h_{i}(\boldsymbol{\theta}(t)) h_{j}(\boldsymbol{\theta}(t)) \mathbf{q}^{\circ T}(t) \mathbf{P}_{i j}^{\circ} \mathbf{q}^{\circ}(t)<0,
$$

where

$$
\begin{gathered}
\mathbf{P}_{i j}^{\circ}=\left[\begin{array}{cc}
\mathbf{S}_{1}\left(\mathbf{A}_{i}-\mathbf{B}_{i} \mathbf{K}_{j} \mathbf{C}\right)+\left(\mathbf{A}_{i}-\mathbf{B}_{i} \mathbf{K}_{j} \mathbf{C}\right)^{T} \mathbf{S}_{1}+\mathbf{X}_{i j} & * \\
\mathbf{P}-\mathbf{S}_{1}+\mathbf{S}_{2}\left(\mathbf{A}_{i}-\mathbf{B}_{i} \mathbf{K}_{j} \mathbf{C}\right) & -2 \mathbf{S}_{2}
\end{array}\right] \\
<0 \\
\mathbf{Z}^{\circ}(\boldsymbol{\theta}(t))=\operatorname{diag}\left[\begin{array}{ll}
\mathbf{Z}(\boldsymbol{\theta}(t)) & \mathbf{0}] \geq 0 .
\end{array}\right.
\end{gathered}
$$

Since $r=m$, it is now possible to set

$$
\mathbf{B}_{i} \mathbf{K}_{j} \mathbf{C}=\mathbf{B}_{i} \mathbf{K}_{j} \mathbf{M M}^{-1} \mathbf{C}=\mathbf{B}_{i} \mathbf{N}_{j} \mathbf{C S}_{1},
$$

where

$$
\mathbf{K}_{j} \mathbf{M}=\mathbf{N}_{j}, \quad \mathbf{M}^{-1} \mathbf{C}=\mathbf{C S}_{1},
$$

and $\mathbf{M} \in \mathbb{R}^{m \times m}$ is a regular square matrix. Substituting (51) into (49) results in

$$
\mathbf{P}_{i j}^{\circ}=\left[\begin{array}{cc}
\boldsymbol{\Phi}_{i j} & * \\
\mathbf{P}-\mathbf{S}_{1}+\mathbf{S}_{2}\left(\mathbf{A}_{i}-\mathbf{B}_{i} \mathbf{N}_{j} \mathbf{C S}_{1}\right) & -2 \mathbf{S}_{2}
\end{array}\right],
$$


where

$$
\boldsymbol{\Phi}_{i j}=\mathbf{X}_{i j}+\mathbf{S}_{1}\left(\mathbf{A}_{i}-\mathbf{B}_{i} \mathbf{N}_{j} \mathbf{C S}_{1}\right)+\left(\mathbf{A}_{i}-\mathbf{B}_{i} \mathbf{N}_{j} \mathbf{C S}_{1}\right)^{T} \mathbf{S}_{1} .
$$

Defining the congruence transform matrix $\mathbf{T}_{c}$ as follows:

$$
\mathbf{T}_{c}=\left[\begin{array}{ll}
\mathbf{S}_{1}^{-1} & \mathbf{S}_{2}^{-1}
\end{array}\right] \text {. }
$$

Premultiplying the left and the right side of (53) and (50) by $\mathbf{T}_{c}$ gives

$$
\begin{aligned}
& {\left[\begin{array}{cc}
\boldsymbol{\Pi}_{i j} & * \\
\mathbf{S}_{2}^{-1} \mathbf{P S}_{1}^{-1}-\mathbf{S}_{2}^{-1}+\left(\mathbf{A}_{i} \mathbf{S}_{1}^{-1}-\mathbf{B}_{i} \mathbf{N}_{j} \mathbf{C}\right) & -2 \mathbf{S}_{2}^{-1}
\end{array}\right]<0} \\
& \operatorname{diag}\left[\begin{array}{ll}
\mathbf{S}_{1}^{-1} \mathbf{Z}(\boldsymbol{\theta}(t)) \mathbf{S}_{1}^{-1} & \mathbf{0}
\end{array}\right] \geq 0,
\end{aligned}
$$

where

$$
\begin{aligned}
\boldsymbol{\Pi}_{i j}= & \mathbf{S}_{1}^{-1} \boldsymbol{\Phi}_{i j} \mathbf{S}_{1}^{-1}=\mathbf{S}_{1}^{-1} \mathbf{X}_{i j} \mathbf{S}_{1}^{-1} \\
& +\left(\mathbf{A}_{i} \mathbf{S}_{1}^{-1}-\mathbf{B}_{i} \mathbf{N}_{j} \mathbf{C}\right)+\left(\mathbf{A}_{i} \mathbf{S}_{1}^{-1}-\mathbf{B}_{i} \mathbf{N}_{j} \mathbf{C}\right)^{T}
\end{aligned}
$$

Thus, using the notations

$$
\mathbf{V}=\mathbf{S}_{1}^{-1}, \quad \mathbf{U}=\mathbf{S}_{2}^{-1}, \quad \mathbf{T}=\mathbf{U P V}, \quad \mathbf{Y}_{i j}=\mathbf{V} \mathbf{X}_{i j} \mathbf{V}
$$

then (56), (58) implies (41).

Since now, $\mathbf{V Z}(\boldsymbol{\theta}(t)) \mathbf{V}$ in (57) and $\mathbf{W Z}(\boldsymbol{\theta}(t)) \mathbf{W}$ in (28), both have the same structure if $\mathbf{V}=\mathbf{W},(57)$ implies (40).

In addition, (52) gives

$$
\mathrm{MC}=\mathrm{CS}_{1}^{-1}=\mathbf{C V}
$$

and since (60) and (30) both have the same structure if $\mathbf{V}=$ W, then (60) implies (42). This concludes the proof.

This principle naturally exploits the affine TS model properties. Introducing the slack matrix variables $\mathbf{U}, \mathbf{V}$ into the LMIs, the system matrices are decoupled from the equivalent Lyapunov matrix T. Note, the above-presented inequalities are linear matrix inequalities, but the equivalent Lyapunov matrix $\mathbf{T}$ is not a symmetric matrix. Introducing a scalar design parameter $\delta>0, \delta \in \mathbb{R}$, Theorem 4 can be modified in the next form.

Corollary 5. If, instead of the notations (56), there are used in (59) the next substitutions

$$
\mathbf{U}=\delta \mathbf{V}, \quad \mathbf{T}=\delta \mathbf{V P V}, \quad \delta>0, \delta \in \mathbb{R} .
$$

It is evident that (53) and (54) with (61) imply

$$
\begin{gathered}
\mathbf{T}=\mathbf{T}^{T}>0, \quad \mathbf{V}=\mathbf{V}^{T}>0 \\
{\left[\begin{array}{cccc}
\mathbf{Y}_{11} & \mathbf{Y}_{12} & \cdots & \mathbf{Y}_{1 s} \\
\mathbf{Y}_{12} & \mathbf{Y}_{22} & \cdots & \mathbf{Y}_{2 s} \\
\vdots & \vdots & \ddots & \vdots \\
\mathbf{Y}_{1 s} & \mathbf{Y}_{2 s} & \cdots & \mathbf{Y}_{s s}
\end{array}\right]>0,} \\
{\left[\begin{array}{c}
\mathbf{A}_{i} \mathbf{V}+\mathbf{V} \mathbf{A}_{i}^{T}-\mathbf{B}_{i} \mathbf{N}_{j} \mathbf{C}-\mathbf{C}^{T} \mathbf{N}_{j}^{T} \mathbf{B}_{i}^{T}+\mathbf{Y}_{i j} \\
\mathbf{T}-\delta \mathbf{V}+\mathbf{A}_{i} \mathbf{V}-\mathbf{B}_{i} \mathbf{N}_{j} \mathbf{C}
\end{array}\right]<0}
\end{gathered}
$$

for $h_{i}(\boldsymbol{\theta}(t)) h_{j}(\boldsymbol{\theta}(t)) \neq 0, i, j=1,2, \ldots, s$.
Thus, the equilibrium of the fuzzy system (5) and (6), controlled by the fuzzy controller (9), is global asymptotically stable if for given positive scalar $\delta>0, \delta \in \mathbb{R}$ there exist positive definite symmetric matrices $\mathbf{T}, \mathbf{V} \in \mathbb{R}^{n \times n}$, and matrices $\mathbf{N}_{j}, \mathbf{Y}_{i j} \in \mathbb{R}^{r \times n}$ such that (62)-(63) hold. Subsequently, the set of control law gain matrices is given by (42)-(43).

Note, (63) represents the set of LMIs only if $\delta$ is a prescribed constant ( $\delta$ can be considered as a tuning parameter). Considering $\delta$ as a LMI variable, (63) represents the set of bilinear matrix inequalities (BMI).

Theorem 6. The equilibrium of the fuzzy system (5) and (6), controlled by the fuzzy controller (9) is global asymptotically stable if there exist positive definite symmetric matrices $\mathbf{U}, \mathbf{V} \in$ $\mathbb{R}^{n \times n}$, a matrix $\mathbf{T} \in \mathbb{R}^{n \times n}$, and matrices $\mathbf{N}_{j}, \mathbf{Y}_{i j} \in \mathbb{R}^{r \times n}$ such that

$$
\begin{aligned}
& \mathbf{U}=\mathbf{U}^{T}>0, \quad \mathbf{V}=\mathbf{V}^{T}>0, \\
& {\left[\begin{array}{cccc}
\mathbf{Y}_{11} & \mathbf{Y}_{12} & \cdots & \mathbf{Y}_{1 s} \\
\mathbf{Y}_{12} & \mathbf{Y}_{22} & \cdots & \mathbf{Y}_{2 s} \\
\vdots & \vdots & \ddots & \vdots \\
\mathbf{Y}_{1 s} & \mathbf{Y}_{2 s} & \cdots & \mathbf{Y}_{s s}
\end{array}\right]>0} \\
& {\left[\begin{array}{cc}
\mathbf{H}_{i i}+\mathbf{H}_{i i}^{T}+\mathbf{Y}_{i i} & * \\
\mathbf{T}-\mathbf{U}+\mathbf{H}_{i i} & -2 \mathbf{U}
\end{array}\right]<0,} \\
& {\left[\begin{array}{cc}
\frac{\mathbf{H}_{i j}+\mathbf{H}_{j i}}{2}+\frac{\left(\mathbf{H}_{i j}+\mathbf{H}_{j i}\right)^{T}}{2}+\frac{\mathbf{Y}_{i j}+\mathbf{Y}_{j i}}{2} & * \\
\mathbf{T}-\mathbf{U}+\frac{\mathbf{H}_{i j}+\mathbf{H}_{j i}}{2} & -2 \mathbf{U}
\end{array}\right]<0}
\end{aligned}
$$

for all $i \in\langle 1,2, \ldots s\rangle, i<j \leq s, i, j \in\langle 1,2, \ldots s\rangle$, respectively, and $h_{i}(\boldsymbol{\theta}(t)) h_{j}(\boldsymbol{\theta}(t)) \neq 0$. Here it is

$$
\mathbf{H}_{i j}=\mathbf{A}_{i} \mathbf{V}-\mathbf{B}_{i} \mathbf{N}_{j} \mathbf{C}
$$

and if the above conditions hold, the set of control law gain matrices is given as in (42)-(43).

Proof. Considering (41) and (67), (48) can be written as

$$
\begin{aligned}
\dot{v}(\mathbf{q}(t))= & \sum_{i=1}^{s} \sum_{j=1}^{s} h_{i}(\boldsymbol{\theta}(t)) h_{j}(\boldsymbol{\theta}(t)) \\
& \times \mathbf{q}^{\circ T}(t) \mathbf{T}_{c}^{-1} \mathbf{T}_{c} \mathbf{P}_{i j}^{\circ} \mathbf{T}_{c} \mathbf{T}_{c}^{-1} \mathbf{q}^{\circ}(t) \\
= & \sum_{i=1}^{s} \sum_{j=1}^{s} h_{i}(\boldsymbol{\theta}(t)) h_{j}(\boldsymbol{\theta}(t)) \mathbf{q}^{\bullet T}(t) \mathbf{P}_{i j}^{\bullet} \mathbf{q}^{\bullet}(t)<0,
\end{aligned}
$$

where with $\mathbf{T}_{c}$ defined in (55), it is

$$
\begin{aligned}
\mathbf{q}^{\bullet}(t) & =\mathbf{T}_{c}^{-1} \mathbf{q}^{\circ}(t) \\
\mathbf{P}_{i j}^{\bullet} & =\mathbf{T}_{c} \mathbf{P}_{i j}^{\circ} \mathbf{T}_{c}=\left[\begin{array}{cc}
\mathbf{H}_{i j}+\mathbf{H}_{i j}^{T}+\mathbf{Y}_{i j} & * \\
\mathbf{T}-\mathbf{U}^{\prime}+\mathbf{H}_{i j} & -2 \mathbf{U}
\end{array}\right]<0 .
\end{aligned}
$$


Permuting the subscripts $i$ and $j$ in (68) gives

$$
\dot{v}(\mathbf{q}(t))=\sum_{i=1}^{s} \sum_{j=1}^{s} h_{i}(\boldsymbol{\theta}(t)) h_{j}(\boldsymbol{\theta}(t)) \mathbf{q}^{\bullet T}(t) \mathbf{P}_{j i}^{\bullet} \mathbf{q}^{\bullet}(t)
$$

and adding (68) and (70) results in

$$
2 \dot{v}(\mathbf{q}(t))=\sum_{i=1}^{s} \sum_{j=1}^{s} h_{i}(\boldsymbol{\theta}(t)) h_{j}(\boldsymbol{\theta}(t)) \mathbf{q}^{\bullet T}(t) \widetilde{\mathbf{P}}_{i j}^{\bullet} \mathbf{q}^{\bullet}(t),
$$

where

$$
\widetilde{\mathbf{P}}_{i j}^{\bullet}=\left[\begin{array}{cc}
\mathbf{H}_{i j}+\mathbf{H}_{j i}+\mathbf{H}_{i j}^{T}+\mathbf{H}_{j i}^{T}+\mathbf{Y}_{i j}+\mathbf{Y}_{j i} & * \\
2 \mathbf{T}-2 \mathbf{U}+\mathbf{H}_{i j}+\mathbf{H}_{j i} & -4 \mathbf{U}
\end{array}\right]<0 .
$$

Rearranging the computation, (71) takes the form

$$
\begin{aligned}
\dot{v}(\mathbf{q}(t))= & \sum_{i=1}^{s} h_{i}^{2}(\boldsymbol{\theta}(t)) \mathbf{q}^{T}(t) \frac{1}{2} \widetilde{\mathbf{P}}_{i i}^{\bullet} \mathbf{q}^{\bullet}(t) \\
& +2 \sum_{i=1}^{s-1} \sum_{j=i+1}^{s} h_{i}(\boldsymbol{\theta}(t)) h_{j}(\boldsymbol{\theta}(t)) \mathbf{q}^{\bullet T}(t) \frac{1}{2} \widetilde{\mathbf{P}}_{i j}^{\bullet} \mathbf{q}^{\bullet}(t),
\end{aligned}
$$

where

$$
\begin{gathered}
\frac{1}{2} \widetilde{\mathbf{P}}_{i i}^{\bullet}=\left[\begin{array}{cc}
\mathbf{H}_{i i}+\mathbf{H}_{i i}^{T}+\mathbf{Y}_{i i} & * \\
\mathbf{T}-\mathbf{U}+\mathbf{H}_{i i} & -2 \mathbf{U}
\end{array}\right]<0 \\
\frac{1}{2} \widetilde{\mathbf{P}}_{i j}^{\bullet}=\left[\begin{array}{cc}
\frac{\mathbf{H}_{i j}+\mathbf{H}_{j i}}{2}+\frac{\left(\mathbf{H}_{i j}+\mathbf{H}_{j i}\right)^{T}}{2}+\frac{\mathbf{Y}_{i j}+\mathbf{Y}_{j i}}{2} & * \\
\mathbf{T}-\mathbf{U}+\frac{\mathbf{H}_{i j}+\mathbf{H}_{j i}}{2} & -2 \mathbf{U}
\end{array}\right]<0
\end{gathered}
$$

and, evidently, (74), (75) imply (65),(66) respectively. This concludes the proof.

\section{Illustrative Example}

The nonlinear dynamics of the hydrostatic transmission was taken from [24] and this model was used in control design and simulation.

The hydrostatic transmission dynamics is represented by a nonlinear fourth-order state-space model as follows.

$$
\begin{aligned}
& \dot{q}_{1}(t)=-a_{11} q_{1}(t)+b_{11} u_{1}(t), \\
& \dot{q}_{2}(t)=-a_{22} q_{2}(t)+b_{22} u_{2}(t), \\
& \dot{q}_{3}(t)=a_{31} q_{1}(t) p(t)-a_{33} q_{3}(t)-a_{34} q_{2}(t) q_{4}(t), \\
& \dot{q}_{4}(t)=a_{43} q_{2}(t) q_{3}(t)-a_{44} q_{4}(t),
\end{aligned}
$$

where $q_{1}(t)$ is the normalized hydraulic pump angle, $q_{2}(t)$ is the normalized hydraulic motor angle, $q_{3}(t)$ is the pressure difference (bar), $q_{4}(t)$ is the hydraulic motor speed ( $\left.\mathrm{rad} / \mathrm{s}\right)$, $p(t)$ is the speed of hydraulic pump ( $\mathrm{rad} / \mathrm{s}), u_{1}(t)$ is the normalized control signal of the hydraulic pump, and $u_{2}(t)$ is the normalized control signal of the hydraulic motor. It is supposed that the external variable $p(t)$, as well as the second state variable $q_{2}(t)$ are measurable. In given working point the parameters are

$$
\begin{gathered}
a_{11}=7.6923 \quad a_{22}=4.5455 \quad a_{33}=7.6054 .10^{-4}, \\
a_{31}=0.7877 \quad a_{34}=0.9235 \quad b_{11}=1.8590 .10^{3}, \text { and } \\
a_{43}=12.1967 \quad a_{44}=0.4143 \quad b_{22}=1.2879 .10^{3} .
\end{gathered}
$$

Since the variables $p(t) \in\langle 105,300\rangle$ and $q_{2}(t) \in\langle 0.0001,1\rangle$ are bounded on the prescribed sectors then vector of the premise variables can be chosen as follows:

$$
\boldsymbol{\theta}(t)=\left[\begin{array}{ll}
\theta_{1}(t) & \theta_{2}(t)
\end{array}\right]=\left[\begin{array}{ll}
q_{2}(t) & p(t)
\end{array}\right] .
$$

Thus, the set of nonlinear sector functions as follows.

$$
\begin{aligned}
& w_{11}\left(q_{2}(t)\right)=\frac{b_{1}-q_{2}(t)}{b_{1}-b_{2}}, \\
& w_{12}\left(q_{2}(t)\right)=\frac{q_{2}(t)-b_{2}}{b_{1}-b_{2}}=1-w_{11}\left(q_{2}(t)\right), \\
& b_{1}=0, b_{2}=1,
\end{aligned}
$$

$$
\begin{aligned}
w_{21}(p(t))=\frac{c_{1}-p(t)}{c_{1}-c_{2}}, & \\
w_{22}(p(t))=\frac{p(t)-c_{2}}{c_{1}-c_{2}} & =1-w_{21}(p(t)), \\
c_{1} & =105, c_{2}=300
\end{aligned}
$$

implies the next set of normalized membership functions as follows:

$$
\begin{aligned}
& h_{1}\left(q_{2}(t), p(t)\right)=w_{11}\left(q_{2}(t)\right) w_{21}(p(t)), \\
& h_{2}\left(q_{2}(t), p(t)\right)=w_{12}\left(q_{2}(t)\right) w_{21}(p(t)), \\
& h_{3}\left(q_{2}(t), p(t)\right)=w_{11}\left(q_{2}(t)\right) w_{22}(p(t)), \\
& h_{4}\left(q_{2}(t), p(t)\right)=w_{12}\left(q_{2}(t)\right) w_{22}(p(t)) .
\end{aligned}
$$

The transformation of nonlinear differential equation systems into a TS fuzzy system in standard form gives

$$
\begin{aligned}
\mathbf{A}_{i} & =\left[\begin{array}{cccc}
-a_{11} & 0 & 0 & 0 \\
0 & -a_{22} & 0 & 0 \\
a_{31} c_{k} & 0 & -a_{31} & -a_{34} b_{l} \\
0 & 0 & a_{43} b_{l} & -a_{44}
\end{array}\right], \quad \mathbf{B}=\left[\begin{array}{cc}
a_{11} & 0 \\
0 & b_{22} \\
0 & 0 \\
0 & 0
\end{array}\right], \\
\mathbf{C}^{T} & =\left[\begin{array}{ll}
0 & 0 \\
1 & 0 \\
0 & 1 \\
0 & 0
\end{array}\right]
\end{aligned}
$$

with the associations

$$
\begin{array}{ll}
i=1 \longleftarrow(l=1, k=1) & i=2 \longleftarrow(l=2, k=1) \\
i=3 \longleftarrow(l=1, k=2) & i=4 \longleftarrow(l=2, k=2) .
\end{array}
$$




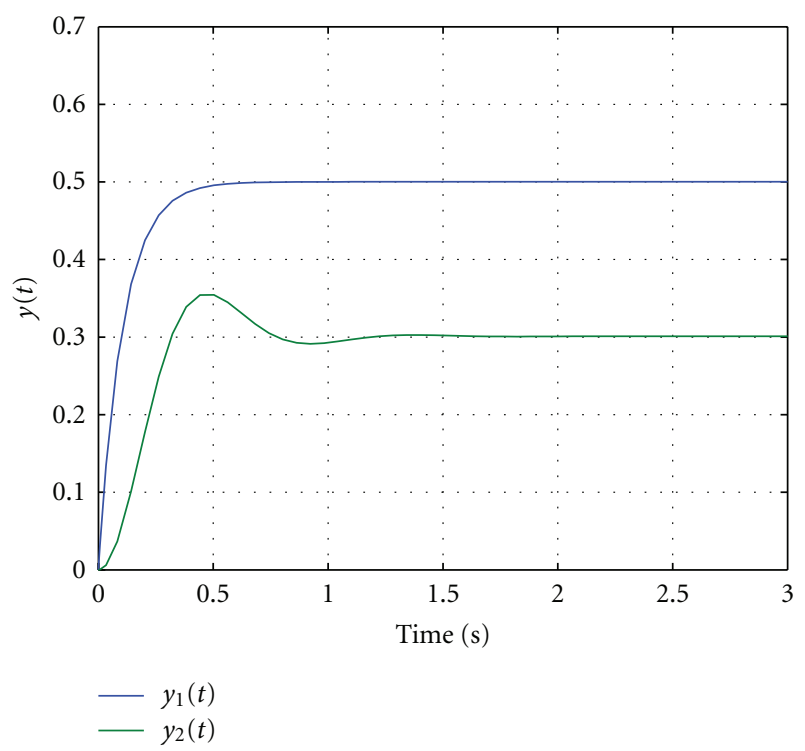

FIGURE 1: System output response.

Thus, solving (62), (63) with respect to the LMI matrix variables $\mathbf{T}, \mathbf{V}, \mathbf{N}_{j}, j=1,2,3,4$, and with $\delta=9$ then, using Self-Dual-Minimization (SeDuMi) package for Matlab $[25,26]$, the feedback gain matrix design problem was feasible with the results

$$
\begin{gathered}
\mathbf{T}=\left[\begin{array}{cccc}
0.0017 & 0.0000 & -0.0223 & 0.0006 \\
0.0000 & 0.9258 & 0.0000 & 0.0000 \\
-0.0223 & 0.0000 & 0.5679 & -0.1464 \\
0.0006 & 0.0000 & -0.1464 & 1.7684
\end{array}\right]>0 \\
\mathbf{V}=\left[\begin{array}{cccc}
0.0001 & 0.0000 & -0.0017 & -0.0001 \\
0.0000 & 0.0481 & 0.0000 & -0.0000 \\
-0.0017 & 0.0000 & 0.0410 & -0.0253 \\
-0.0001 & 0.0000 & -0.0253 & 0.2027
\end{array}\right]>0 \\
\mathbf{K}_{i}=\left[\begin{array}{lll}
0.0000 & 0.0002 \\
0.0047 & 0.0000
\end{array}\right], \quad i=1,2,3,4
\end{gathered}
$$

which rise up a stable set of closed-loop subsystems. It can be seen that with an enough precision the used design conditions imply the approximately equal control gain matrices. Comparing with design methods proposed in [17], the fuzzy control is so less conservative.

The conditions in simulations were specified for the system in the forced regime, where

$$
\begin{gathered}
\mathbf{u}(t)=\sum_{j=1}^{s} h_{j}(\boldsymbol{\theta}(t))\left(-\mathbf{K}_{j} \mathbf{C q}(t)+\mathbf{W W}(t)\right), \\
\mathbf{W}=\left[\begin{array}{ll}
0.0000 & 0.0002 \\
0.0073 & 0.0000
\end{array}\right], \quad \mathbf{W}(t)=\left[\begin{array}{l}
0.5 \\
0.3
\end{array}\right], \\
\mathbf{q}(0)=\mathbf{0}, \quad p(t)=105 .
\end{gathered}
$$

Figure 1 shows the simulation result for the system with zero initial state.

\section{Concluding Remarks}

New approach for output feedback control design, taking into account the output matrix of the system model and the affine properties of the TS model structure, is presented in this paper. Applying the fuzzy output control scheme relating to the parallel distributed output compensators and introducing the slack matrices into an enhanced Lyapunov inequality, the method significantly reduces the conservativeness in the control design conditions. By the proposed procedure, strictly decoupling a Lyapunov matrix and the system parameter matrices in the LMIs, the control problem is parameterized in such LMI structure which admit more freedom in guaranteeing the output feedback control performances.

Sufficient conditions of the controller existence, manipulating the global stability of the system, imply the control structure which stabilizes the nonlinear system in the sense of Lyapunov, and the design of controller parameters directly from these conditions is a solved numerical problem. An additional benefit of the method is that controllers use minimum feedback information with respect to desired system output and the approach is flexible enough to allow the inclusion of additional design conditions such as fuzzy Lyapunov functions. The validity and applicability of the approach is demonstrated through a numerical design example.

\section{Acknowledgments}

The work presented in this paper was supported by VEGA, Grant Agency of Ministry of Education, and Academy of Science of Slovak Republic under Grant no. 1/0256/11. This support is very gratefully acknowledged.

\section{References}

[1] T. Takagi and M. Sugeno, "Fuzzy identification of systems and its applications to modeling and control," IEEE Transactions on Systems, Man and Cybernetics, vol. 15, no. 1, pp. 116-132, 1985.

[2] H. O. Wang, K. Tanaka, and M. F. Griffin, "An approach to fuzzy control of nonlinear systems: Stability and design issues," IEEE Transactions on Fuzzy Systems, vol. 4, no. 1, pp. 14-23, 1996.

[3] K. M. Passino and S. Yurkovich, Fuzzy Control, Addison-Wesley Longman, Berkeley, Calif, USA, 1998.

[4] M. Johansson, A. Rantzer, and K. E. Årzén, "Piecewise quadratic stability of fuzzy systems," IEEE Transactions on Fuzzy Systems, vol. 7, no. 6, pp. 713-722, 1999.

[5] K. Tanaka and H. O. Wang, Fuzzy Control Systems Design and Analysis. A Linear Matrix Inequality Approach, John Wiley \& Sons, New York, NY, USA, 2001.

[6] F. Khaber, K. Zehar, and A. Hamzaoui, "State feedback controller design via Takagi-Sugeno fuzzy model: LMI approach," International Journal of Computational Intelligence, vol. 2, no. 3, pp. 148-153, 2005.

[7] K. Michels, F. Klawonn, R. Kruse, and A. Nurnberger, Fuzzy Control. Fundamentals, Stability and Design of Fuzzy Controllers, Springer, Berlin, Germany, 2006.

[8] I. Abdelmalek, N. Goléa, and M. L. Hadjili, "A new fuzzy Lyapunov approach to non-quadratic stabilization of TakagiSugeno fuzzy models," International Journal of Applied Mathematics and Computer Science, vol. 17, no. 1, pp. 39-51, 2007. 
[9] D. Krokavec and A. Filasova, "Optimal fuzzy control for a class of nonlinear systems," Mathematical Problems in Engineering, vol. 2012, Article ID 481942, 29 pages, 2012.

[10] J. Pan, S. Fei, Y. Ni, and M. Xue, "New approaches to relaxed stabilization conditions and $H_{\infty}$ control designs for T-S fuzzy systems," Journal of Control Theory and Applications, vol. 10, no. 1, pp. 82-91, 2012.

[11] M. P. A. Santim, M. C. M. Teixeira, W. A. de Souza, R. Cardim, and E. Assunao, "Design of a Takagi-Sugeno fuzzy regulator for a set of operation points," Mathematical Problems in Engineering, Article ID 731298, 17 pages, 2012.

[12] B. Boyd, L. El Ghaoui, E. Peron, and V. Balakrishnan, Linear Matrix Inequalities in System and Control Theory, SIAM Society for Industrial and Applied Mathematics, Philadelphia, Pa, USA, 1994.

[13] X. Liu and Q. Zhang, "New approaches to $H_{\infty}$ controller designs based on fuzzy observers for T-S fuzzy systems via LMI," Automatica, vol. 39, no. 9, pp. 1571-1582, 2003.

[14] S. K. Nguang and P. Shi, " $H_{\infty}$ fuzzy output feedback control design for nonlinear systems: an LMI approach," IEEE Transactions on Fuzzy Systems, vol. 11, no. 3, pp. 331-340, 2003.

[15] S. W. Kau, H. J. Lee, C. M. Yang, C. H. Lee, L. Hong, and C. H. Fang, "Robust $H_{\infty}$ fuzzy static output feedback control of TS fuzzy systems with parametric uncertainties," Fuzzy Sets and Systems, vol. 158, no. 2, pp. 135-146, 2007.

[16] B. S. Chen, C. S. Tseng, and H. J. Uang, "Mixed $H_{2} / H_{\infty}$ fuzzy output feedback control design for nonlinear dynamic systems: an LMI approach," IEEE Transactions on Fuzzy Systems, vol. 8, no. 3, pp. 249-265, 2000.

[17] M. Chadli, D. Maquin, and J. Ragot, "Static output feedback for Takagi-Sugeno systems: an LMI approach," in Proceedings of the 10th Mediterranean Conference on Control and Automation (MED '02), Lisbon, Portugal, July 2002.

[18] H. L. Jhi and C. S. Tseng, "Robust static output feedback fuzzy control design for nonlinear discrete-time systems with persistent bounded disturbances," International Journal of Fuzzy Systems, vol. 14, no. 1, pp. 131-140, 2012.

[19] E. S. Tognetti, R. C. L. F. Oliveira, and P. L. D. Peres, "Improved stabilization conditions for Takagi-Sugeno fuzzy systems via fuzzy integral Lyapunov functions," in Proceedings of the American Control Conference (ACC '11), pp. 4970-4975, San Francisco, Calif, USA, June 2011.

[20] D. Krokavec and A. Filasova, "Stabilizing fuzzy static output control for a class of nonlinear systems," in Proceedings of the 16th IEEE International Conference on Intelligent Engineering Systems (INES '12), pp. 285-290, Lisbon, Portugal, June 2012.

[21] D. Huang and S. K. Nguang, "Static output feedback controller design for fuzzy systems: an ILMI approach," Information Sciences, vol. 177, no. 14, pp. 3005-3015, 2007.

[22] W. M. Haddad and V. Chellaboina, Nonlinear Dynamical Systems and Control. A Lyapunov-Based Approach, Princeton University Press, Princeton, NJ, USA, 2008.

[23] E. Kim and H. Lee, "New approaches to relaxed quadratic stability condition of fuzzy control systems," IEEE Transactions on Fuzzy Systems, vol. 8, no. 5, pp. 523-534, 2000.

[24] P. Gerland, D. Groß, H. Schulte, and A. Kroll, "Robust adaptive fault detection using global state information and application to mobile working machines," in Proceedings of the 1st Conference on Control and Fault-Tolerant Systems (SysTol '10), pp. 813-818, Nice, France, October 2010.

[25] D. Krokavec and A. Filasova, Discrete-Time Systems, Elfa, Kosice, Slovakia, 2008.

[26] D. Peaucelle, D. Henrion, Y. Labit, and K. Taitz, USer's Guide for SeDuMi Interface 1. 04, LAAS-CNRS, Toulouse, France, 2002. 

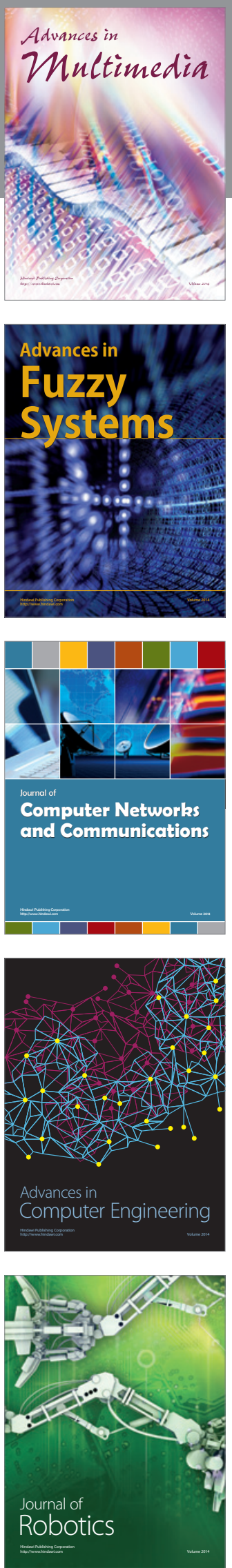

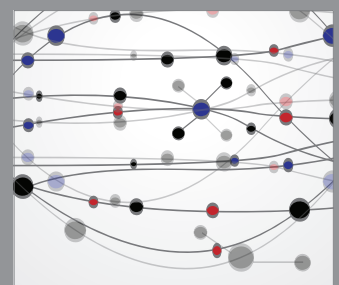

The Scientific World Journal
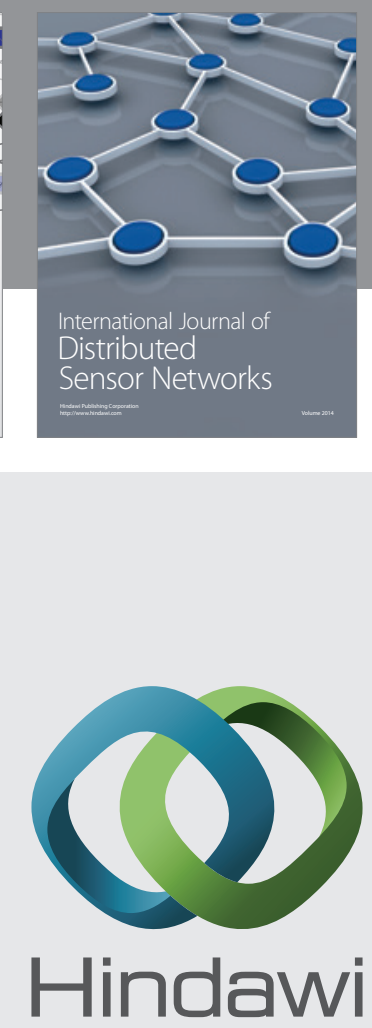

Submit your manuscripts at

http://www.hindawi.com
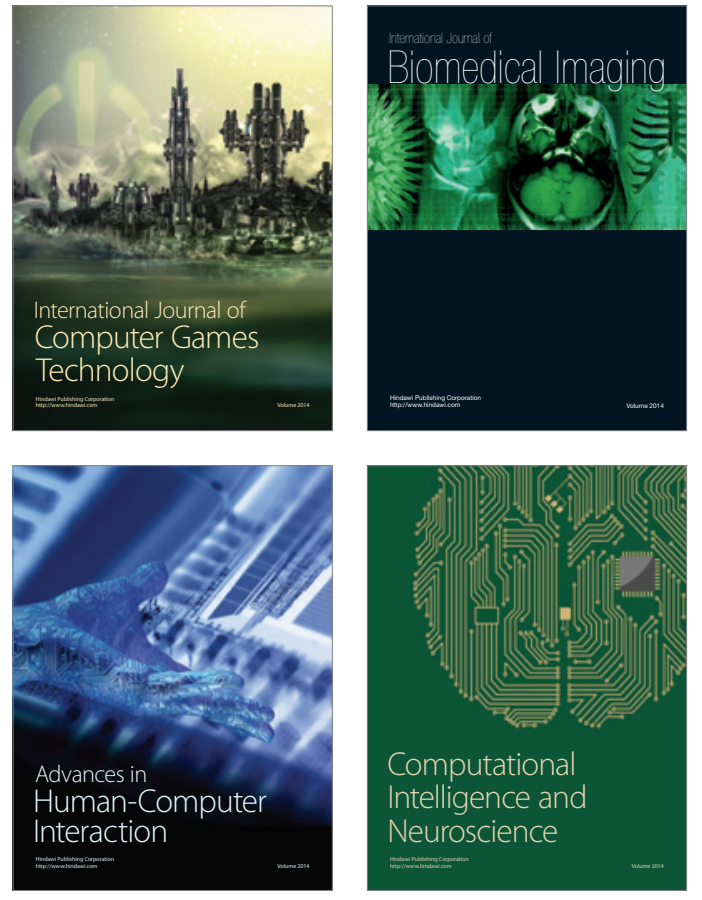
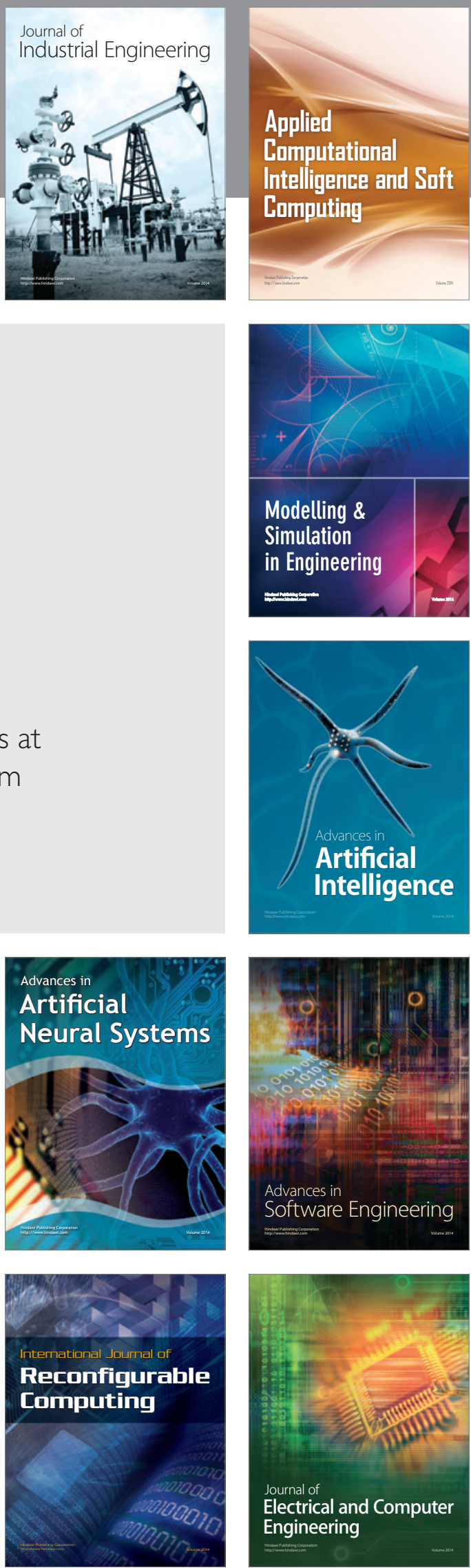